\section{Hochdosiertes Cytarabin bei Mantelzelllymphom}

Die Langzeitprognose des Mantelzelllymphoms (MCL) ist ungünstig. In einer Phase-III-Studie wurde die Zugabe von hochdosiertem Cytarabin zur Immunchemotherapie vor autologer Stammzelltransplantation (ASCT) untersucht.

O bwohl sich das mediane Gesamtüberleben (OS) beim MCL in den letzten Jahrzehnten auf 4 bis 5 Jahre verdoppelt hat, bleibt die Krankheit unheilbar. Selbst wenn Rituximab zur Chemotherapie hinzugefügt wird (R-CHOP), liegt die Rate kompletter Remissionen (CR) derzeit bei unter $50 \%$, die Zeit bis zum Progress beträgt oft unter 2 Jahre.

An der randomisierten Phase-III-Studie des European MCL Network nahmen 497 Patienten (median 55 Jahre) mit unbehandeltem MCL im Stadium II-IV teil. Sie wurden auf 6 Zyklen R-CHOP, gefolgt von myeloablativer Radiochemotherapie und ASCT (Kontrollgruppe) oder 6 Zyklen alternierend R-CHOP oder R-DHAP (Rituximab plus Dexamethason, hochdosiertes Cytarabin und
Cisplatin), gefolgt von einem Regime mit hochdosiertem Cytarabin und ASCT (Cytarabin-Gruppe) randomisiert. Primärer Endpunkt war die Zeit bis zum Therapieversagen nach mindestens 4 Induktionszyklen, Progression oder Tod.

Nach median 6,1 Jahren war die mediane Zeit bis zum Therapieversagen in der Cytarabin-Gruppe signifikant länger (9,1 vs. 3,9 Jahre). Die Rate für das 5-Jahres-OS betrug in der Cytarabin-Gruppe $65 \%$, in der Kontrollgruppe $40 \%$ (Hazard Ratio 0,56; p = 0,038). Nach Induktion erreichten $90 \%$ der Patienten in der Kontroll- und 94\% in der Cytarabin-Gruppe eine Remission $(p=0,14), 25$ versus $38 \%$ davon eine CR $(p=0,0016)$. Nach der ASCT waren in beiden Gruppen die Raten für das An- sprechen (97 vs. $98 \%)$ und eine CR (61 vs. $63 \%$ ) vergleichbar. Während der Induktion litten Patienten der CytarabinGruppe häufiger unter hämatologischen Nebenwirkungen vom Grad 3/4 (Anämie: 29 vs. $8 \%$, Thrombozytopenie: 73 vs. $9 \%$, febrile Neutropenie: 17 vs. $8 \%$ ) und Nierenfunktionsstörungen vom Grad 1/2 (erhöhtes Kreatinin: 43 vs. $10 \%)$. Die Zahl der Todesfälle im Zusammenhang mit der ASCT war in beiden Gruppen vergleichbar (je 3,4\%).

Fazit: Nach Ansicht der Forscher sollte bei Patienten mit MCL unter 65 Jahren eine Immunochemotherapie, die hochdosiertes Cytarabin enthält, vor der ASCT zum Standard werden. Zudem sei es ratsam, die minimale Resterkrankung zu ermitteln.

Kathrin von Kieseritzky

Hermine $\mathrm{O}$ et al. Addition of high-dose cytarabine to immunochemotherapy before autologous stem-cell transplantation in patients aged 65 years or younger with mantle cell lymphoma (MCL Younger): a randomised, open-label, phase 3 trial of the European Mantle Cell Lymphoma Network. Lancet. 2016388(10044):565-75.

\section{Körperliche Fitness korreliert mit Erfolg der BMT}

\section{Derzeit stützt sich die Risikobeurteilung bei Knochenmarktransplantation (BMT) vor allem auf klinische, vom Arzt erhobene und krankheitsbezogene Variablen. Obwohl Angaben des Patienten selbst („patient reported outco- mes"; PRO) bei vielen Malignomen eine Korrelation mit dem Mortalitätrisiko zeigen, werden sie bei der Transplantation bislang weniger berücksichtigt.}

U ntersucht wurde, ob vor der Transplantation erhobene Gesundheitsaussagen des Patienten, speziell zu körperlichen Funktionen, eine Prognose des Transplantationserfolgs erlauben.

Dazu nutzten Forscher die Daten einer breit angelegten Primärstudie in den USA zum Effekt eines körperlichen Trainings. Erfasst wurden die Daten von 336 Patienten mit allogener (alloSCT) und 310 mit autologer Stammzelltransplantation (ASCT). Mit zwei Domänen des SF-36-Fragebogens - der PCS (Physical Component Summary) und der MCS (Mental Component Summary) - wurden die PRO vor und nach BMT erfasst und mit Gesamtüberleben und krankheitsspezifischem Überleben korreliert.
Bei alloSCT war der vor Transplantation erhobene PCS, der körperliche Fitness und allgemeine Gesundheit aus $\mathrm{Pa}$ tientensicht spiegelt, ein unabhängiger Prädiktor für das Gesamtüberleben (Hazard Ratio 1,40; p < 0,001); damit war er vergleichbar mit anderen derzeit genutzten Indizes. Die Überlebenswahrscheinlichkeit nach 1 Jahr betrug für die 1., 2., 3. und 4. Quartile des Ausgangs-PCS $50 \%, 65 \%$, $75 \%$ und $83 \%$. Eine frühe Abnahme desr PCS korrelierte mit höherer Gesamt- und krankeitsspezifischer Mortalität.

Wahrscheinlich aufgrund der geringeren Morbidität und Mortalität ergab sich für Patienten mit autoSCT weder für klinische Variablen wie Alter, Pri- märerkrankung, Stadium der Erkrankung etc. noch für PCS oder MCS eine signifikante Korrelation zum Überleben.

Die PRO zur mentalen Gesundheit, erfasst mit dem MCS, waren in der untersuchten Kohorte nicht signifikant mit dem Überleben nach Transplantation assoziiert. Bessere Strategien zur Risikoadaption könnten für Patienten vor BMT von großem Nutzen sein und Patientenselektion und -beratung beeinflussen. Auch zur Beurteilung von Studienergebnissen oder zum Vergleich zentrumsspezifischer Daten zum Transplantationserfolg wäre es wichtig, das Risiko der Patienten möglichst genau abzuschätzen.

Fazit: PRO haben das Potenzial, die Prognoseeinschätzung vor BMT zu verbessern. Die regelmäßige vorherige Erhebung wird empfohlen. Brigitte Schalhorn

Wood WA et al. Patient-reported physical functioning predicts the success of hematopoietic cell transplantation (BMT CTN 0902). Cancer. 2016;122(1):91-8. 